\title{
Erratum to: Energy-aware analysis of electrically long high speed I/O links
}

\author{
Sebastian Müller • Xiaomin Duan • Christian Schuster
}

Published online: 16 November 2012

(C) Springer-Verlag Berlin Heidelberg 2012

\section{Erratum to: Comput Sci Res Dev}

\section{DOI 10.1007/s00450-012-0228-y}

Unfortunately, the first part of the expression for the voltage transfer function in Eq. (3) is missing in the original publication. Correctly, the equation should read

$H=\frac{V_{1}}{V_{2}}=A-\frac{\left(A \cdot Z_{T}+B\right) \cdot\left(A / Z_{R}+C\right)}{\left(A / Z_{R}+C\right) \cdot Z_{T}+B / Z_{R}+D}$.

All figures and results in the original publication have been produced based on the correct equation.
Furthermore, we would like to mention that Eq. (1) should be changed to

$$
\begin{aligned}
\left(\begin{array}{l}
V_{1} \\
I_{1}
\end{array}\right) & =\left[\begin{array}{cc}
1 & 0 \\
\frac{1}{Z_{R}} & 1
\end{array}\right] \cdot\left[\begin{array}{cc}
A & B \\
C & D
\end{array}\right] \cdot\left[\begin{array}{cc}
1 & Z_{T} \\
0 & 1
\end{array}\right]\left(\begin{array}{c}
V_{2} \\
-I_{2}
\end{array}\right) \\
& =\left[\begin{array}{cc}
A & A \cdot Z_{T}+B \\
\frac{A}{Z_{R}}+C & \left(\frac{A}{Z_{R}}+C\right) \cdot Z_{T}+\frac{B}{Z_{R}}+D
\end{array}\right]\left(\begin{array}{c}
V_{2} \\
-I_{2}
\end{array}\right)
\end{aligned}
$$

to be consistent with the current direction defined in Fig. 2(b), although the original equation is correct in itself.

The online version of the original article can be found under doi:10.1007/s00450-012-0228-y. 